\title{
ANÁLISE ESPECTRAL DE EDIFICAÇÃO HISTÓRICA PARA CARACTERIZAÇÃO ESTRUTURAL SOB AÇÕES SÍSMICAS
}

\author{
FREIRES PINTO, MARCELO \\ Estudante \\ LAREB - UFC, Campus Russas \\ Ceará; Brasil \\ marcelofreires18@gmail.com
}

\author{
MINEIRO A. NETO, FRANCISCO \\ Estudante \\ LAREB - UFC, Campus Russas \\ Ceará; Brasil \\ mineironeto1@gmail.com
}

\author{
ISAAC D. PEREIRA, PEDRO \\ Estudante \\ LAREB - UFC, Campus Russas \\ Ceará; Brasil \\ isaacufcrussas@gmail.com
}

\author{
NILTON L. SOUSA, ISRAEL \\ Estudante \\ LAREB - UFC, Campus Russas \\ Ceará; Brasil \\ israelnlsousa@gmail.com
}

\author{
FERNANDES T. MESQUITA, ESEQUIEL \\ Professor, PhD. \\ LAREB - UFC, Campus Russas \\ Ceará; Brasil \\ emesquita@ufc.br
}

\section{RESUMO}

A progressiva evolução técnico-cietífica que envolve a engenharia patrimonial é associada a crescente consciência humana da necessidade de resguardar bens materiais remanescentes, sob uma perspectiva de desenvolvimento apta a absorver técnicas e especialidades de diferentes áreas. Nesse quesito, a sismicidade regional prevista pela caracterização do espectro de resposta confere informações a respeito das acelerações espectrais de projeto que uma região é sujeitada, oportunizando o uso desses dados em análises estruturais de edificações históricas sob cenários de sismicidade acentuada. Ao passo disso, o presente trabalho objetiva caracterizar uma edificação de apoio à construção da barragem do açude Cedro, projetada e construída como alternativa ao combate à seca e contenção de movimentos migratórios, cabendo a edificação em questão, abrigar equipamentos e maquinários entre o final do século XIX e XX. Para isso, recorreu-se a cofecção de um modelo tridimensional via Método dos Elementos Finitos (MEF), para posteriores simulações numéricas no software ANSYS, versão 17.1., que possibilitou a extração de informações técnicas pertinentes aos mecanismos de resposta estrutural sob variáveis de tesões e deslocamentos. Os resultados obtidos permitiram inferir a respeito da converção de esforços oriundos de ações sísmicas em mecanismos danosos nessa edificação e identificar regiões de maior suscetibilidade interventiva, com contribuições ao resguardo e a preservação da identidade sociocultural.

Palavras-chave: Espectro de Resposta, Patrimônio, Caracterização Estrutural, MEF, Sismos.

\begin{abstract}
The progressive technical-scientific evolution that involves heritage engineering is associated with the growing human awareness of the need to safeguard remaining material goods, from a development perspective able to absorb techniques and specialties from different areas. In this regard, the regional seismicity predicted by the response spectrum characterization provides information about the project spectral accelerations that a region is subjected to, allowing the use of this data in structural analysis of historical buildings under accentuated seismicity scenarios. In the meantime, the present work aims to characterize a building that supports the construction of the Cedro Dam, designed and built as an alternative to the fight against drought and the containment of migratory movements. 19th and 20th century. The results obtained allowed us to infer about the conversion of efforts from seismic actions into harmful mechanisms in this building and to identify regions of greater intervention susceptibility, with contributions to the protection and preservation of the socio-cultural identity of those belonging to the conjuncture.
\end{abstract}

Keywords: Response Spectrum, Heritage, Structural Characterization, FEM, Earthquakes.

\section{INSTRODUÇÃO}

Localizado a seis quilômetros da cidade de Quixadá-CE, as obras do Açude Cedro foram concluídas em 1906 e englobam de cinco barragens, estradas de acesso, obras de apoio e uma rede de irrigação que abastecia uma área de 2.000 hectares, sendo considerado a primeira grande obra pública edificada no país e um marco nas obras de combate às secas. Segundo Pereira (2010), nesse período, foram erguidos em Quixadá galpões de beneficiamento do algodão e casas comerciais que 
atendiam as demandas de produtos e serviços da crescente população e que eram escoados para outras partes do Estado. Este açude é um exemplo de representação da ocupação e transformação da paisagem de uma porção do semiárido brasileiro (SILVA, 2017).

Diante a relevância dos trabalhos desenvolvidos no entorno do açude, surge a necessidade de resguardar a integridade desses bens como herança comum para gerações posteriores, de modo a preservar a integridade histórica e cultural associada as construções. A Carta de Cracóvia (2000), reforça que cada comunidade deve desenvolver uma consciência e um conhecimento da importância de preservação do patrimônio construído, pois eles são a identidade cultural para a população.

Nesse sentido, Roca (2010) afirma, que os estudos sobre a conservação e a segurança estrutural do patrimônio histórico edificado, recorrendo-se a análise estrutural via MEF como meio de análise, são ferramentas de grande valia na avaliação destas estruturas pois, permitem conhecer o estado geral das solicitações que são impostas à estrutura, sejam elas por cargas permanentes, cargas acidentais ou cargas excepcionais.

Em síntese, a edificação abordada no desenvolvimento do trabalho trata de um galpão edificado com predominância do emprego de pedra e cal, onde foram abrigadas máquinas utilizadas na construção do açude, servindo também de oficina e almoxarifado (Figura1).

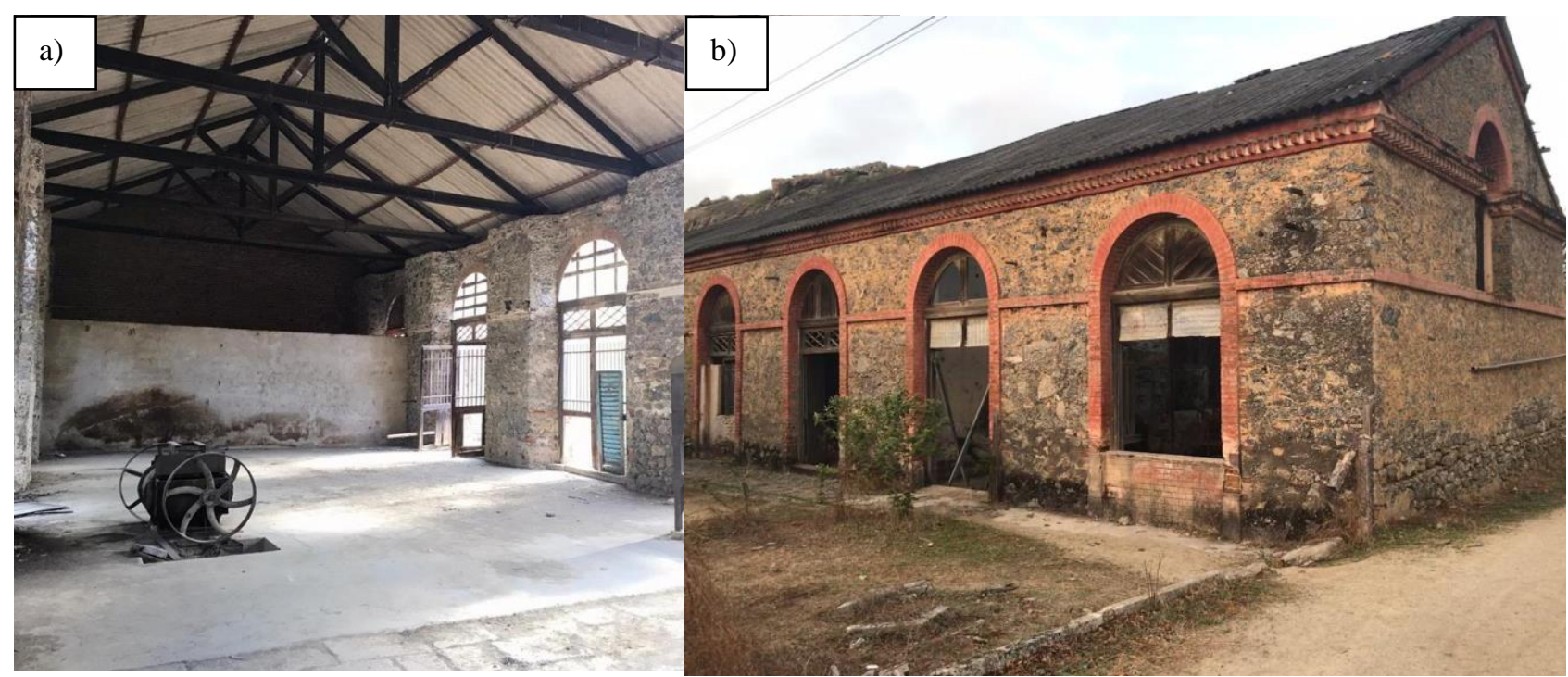

Figura 1: Vistas interna (a) e externa (b) da edificação

No tocante, com o objetivo de caracterizar o comportamento global da edificação, foram confeccionados modelos numéricos tridimensionais recorrendo ao Método dos Elementos Finitos (MEF), com uso do software ANSYS, versão 17.1. Ademais, a formulação do espectro de resposta para a região possibilitou simular o comportamento estrutural sob ações sísmicas, apresentando resultados em variáveis de deslocamento e tensões equivalentes de Von Mises. Desse modo, o presente trabalho vem a contruibuir para publicitar informações que tratam da caracterização e prevenção de danos em construções que compõem o patrimônio histórico nacional.

\section{MODELAGEM NUMÉRICA}

\subsection{Modelo numérico}

Para Lourenço (2002), a micro-modelação é aplicável quando se pretende averiguar com grande detalhe o comportamento localizado da alvenaria e a macro-modelação quando a estrutura é composta por paredes com dimensões tais que a tensão ao longo do elemento pode ser considerada uniforme. Nesse sentido, segundo Peleteiro (2002), cada modelagem apresenta vantagens e desvantagens, a sua escolha depende somente do objetivo a ser alcançado. 
Logo, constatou-se que o nível de precisão requerido para o desenvolvimento da análise global da estrutura torna viável prosseguir a confecção do modelo em elementos finitos com base nos pressupostos da macro-modelagem, nos quais os elementos que compõem a alvenaria podem ser aproximados para um único material com propriedades específicas.

Por meio de lavamentos geométricos, foram obtidas informações que possibilitaram confeccionar um modelo tridimensional que representasse as alvenarias que desempenham função estrutural com máximo de idoneidade (Figura 2). Pode-se observar q ue o telhado não foi modelado para que sua exclusão no modelo acarretasse em simplificações e evitasse possíveis problemas durante a discretização da malha.
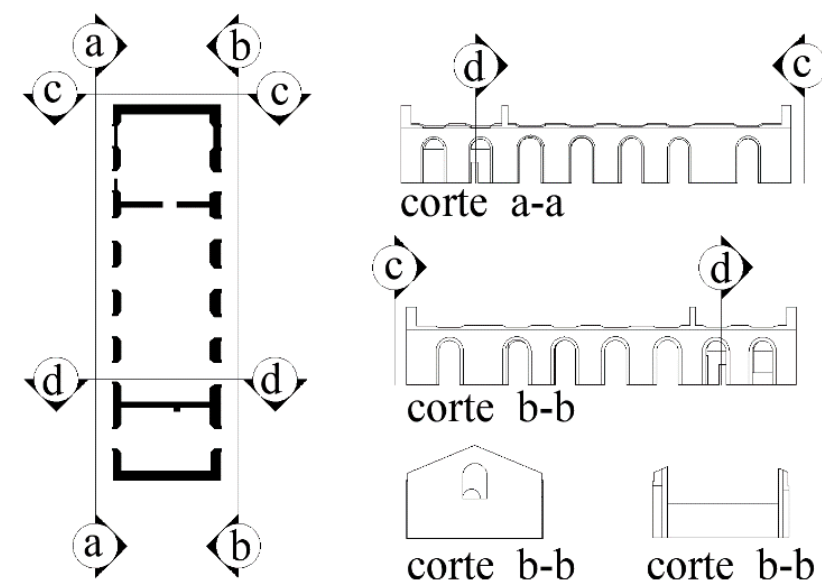

Figura 2: Representação do levantamento geométrico da edificação

Ainda, optou-se por adicionar elementos cerâmicos existentes em regiões próximas os arcos sob a justificativa da presença desses elementos influenciar no comportamento estrutural original, caracterizando a estrutura sob a perspectiva de dois macro-elementos, granítico argamassado e cerâmico argamassado. Segundo Nunes (2013), isso implica que cada macroelemento apresentará, à custa de leis constitutivas pré-estabelecidas, forças e deformações generalizadas, que por sua vez acarretarão uma representação mais rigorosa do comportamento do material obtendo-se resultados com maior nível de idoneidade.

Por fim, o resultado foi um modelo composto pelo elemento SOLID 187, que contem 10 nós com três graus de liberdade para cada nó, adequado para malhas irregulares. Com propriedades que permitem simular grandes deflexões, fluência, rigidez, hiperelasticidade e plasticidade, o resultado final totalizou 697.330 elementos e 120.095 nós.

Elementos Cerâmicos

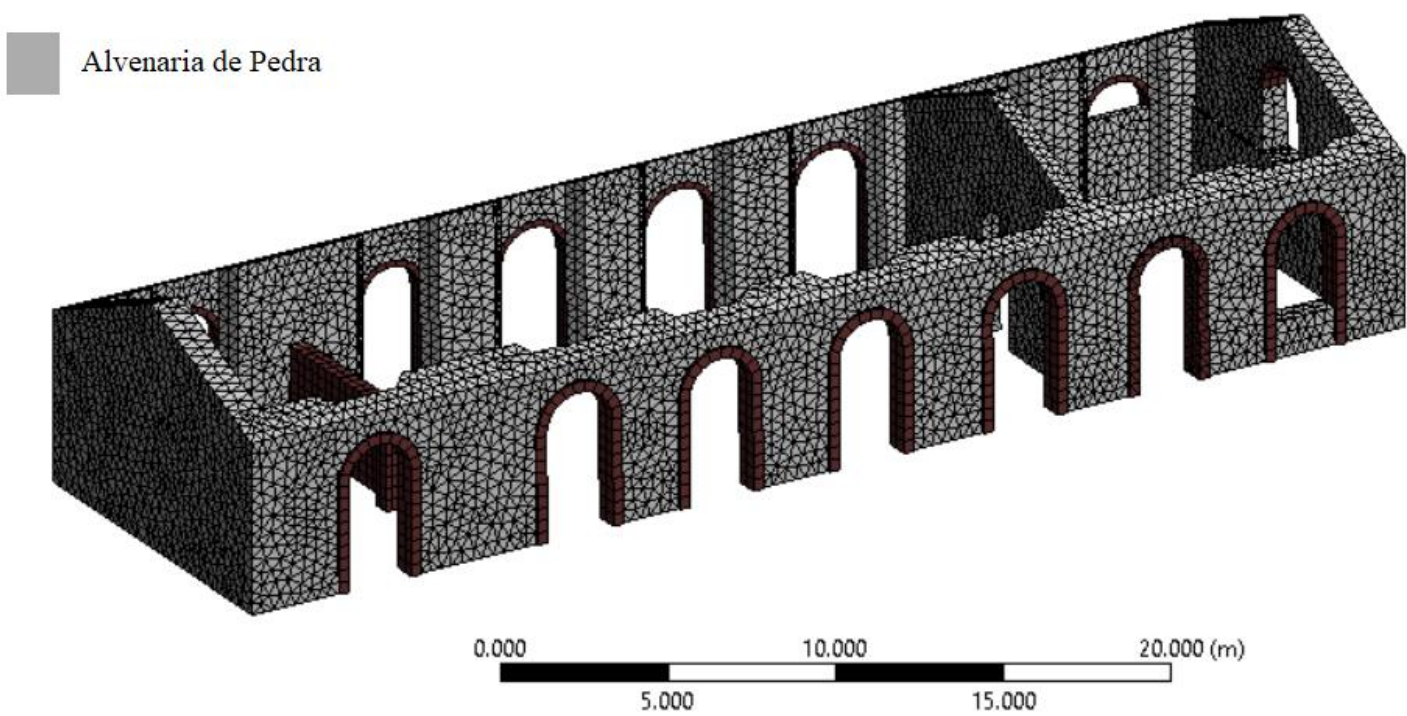

Figura 3: Modelo numérico discretizado em malha de elementos finitos 


\subsection{Propriedades e carregamentos atribuídos ao modelo}

Para o desenvolvimento de simulações numéricas, faz-se necessário caracterizar os materiais que compõem a estrutura da edificação, bem como quantificar as solicitações atuantes, de modo que englobem variáveis mais próximas da realidade. Isso se faz necessário devido a natureza das edificações que compõem o patrimônio histórico, com exigências de durabilidade e desempenho que devem ser garantidas sem que façam necessárias ações interventivas ou quaisquer alterações físicas com divergências das características originais da edificação.

Desse modo, recorreu-se a literatura existente para caracterização dos materiais e quantificação das ações atuantes. O Módulo de Elasticidade (E) considera NCT(MIT,2018) e Brandão(2017), o Peso Específico (w) e a Resistência a Compressão (fc) e foram estimados com base na NCT(MIT,2018), ao passo que a Resistência a Tração (ft) foi obtida com base nos trabalho de Brandão(2017). Por fim, o Coeficiente de Poison (v) foi estimado sob as considerações dos trabalhos de Brandão (2017), Branco (2007) e Frazão (2013).

Tabela 1 - Propriedades mecânicas atribuídas ao modelo numérico

\begin{tabular}{c|c|c|c}
\hline Propriedade & Alvenaria de Pedra & Elemento cerâmico & Referência \\
\hline $\mathrm{E}(\mathrm{GPa})$ & 4,75 & 1,7 & $\begin{array}{c}\text { NCT (MIT,2018) e Brandão } \\
(2017)\end{array}$ \\
\hline $\mathrm{w}\left(\mathrm{KN} / \mathrm{m}^{3}\right)$ & 22 & 18 & NCT (MIT,2018) \\
\hline $\mathrm{Fc}(\mathrm{Mpa})$ & 4,75 & 32 & NCT (MIT,2018) \\
\hline $\mathrm{Ft}(\mathrm{Mpa})$ & 0,24 & 0,16 & Brandão (2017) \\
\hline $\mathrm{V}$ & 0,2 & 0,2 & $\begin{array}{c}\text { Brandão (2017), Branco (2007) } \\
\text { e Frazão (2013) }\end{array}$ \\
\hline
\end{tabular}

Referente aos carregamentos, visto que o telhado não foi modelado, a adição de esforços se fez necessária para que não houvesse total emissão das ações que o madeiramento exerce na estrutura. Assim, seguiu-se as recomendações de Brandão(2017), que considera Branco(2007), Neves(2008) e a NBR 6120(ABNT, 1980), para estimativa dos elementos de coberta (ripas, telhas, caibros e treliças), adotando $1,3 \mathrm{KN} / \mathrm{m}^{2}$ e uma sobrecarga de $0,5 \mathrm{KN} / \mathrm{m}^{2}$. Ainda seguindo as recomendações do autor, foi acrescido $50 \%$ do valor do montante já obtido, totalizando um carregamento de $2,7 \mathrm{KN} / \mathrm{m}^{2}$.

Tabela 2 - Carregamentos utilizados na simulação

\begin{tabular}{c|c|c}
\hline Carregamento & Representação & Referência \\
\hline $1,3 \mathrm{KN} / \mathrm{m}^{2}$ & Ripas, telhas, caibros e treliças & $\begin{array}{c}\text { Brandão (2017), com } \\
\text { considerações de Branco(2007), } \\
\text { Neves(2008) e NBR 6120 } \\
\text { (ABNT,1980) }\end{array}$ \\
\hline $\begin{array}{c}\text { Acréscimo de } 50 \% \text { no } \\
\text { montante obtido }\end{array}$ & Sobrecarga & Brandão(2017) \\
\hline
\end{tabular}

\section{RESULTADOS}

\subsection{Espectro de resposta}

No que tratou da caracterização do espectro de resposta para a localidade em que a edificação se encontra, seguiu-se os parâmetros propostos pela NBR 15421(ABNT, 2006), que faz uso do zoneamento sísmico da região para estimativa de ações de cálculo. Com localização sob a Zona 1, as acelerações características de projeto assumem magnitude de até $0,05 \mathrm{~g}$ e o espectro de resposta de projeto para acelerações horizontais pode ser simplificado a uma resposta elástica com uma fração de amortecimento crítico igual a 5\%, em um sistema de um único grau de liberdade.

Ainda, as acelerações espectrais de projeto (ags0 e ags1) foram definidas com base no uso de fatores de amplificação (Ca e $\mathrm{Cv}$ ) e a aceleração sísmica horizontal(ag). Com isso, o espectro de resposta de projeto $(\mathrm{Sa}(\mathrm{T}))$ passa a ser definido com base na relação período natural (T), sob aplicação nas Equações 1,2 e 3.

$$
\mathrm{Sa}(\mathrm{T})=\operatorname{ags} 0(18,75 \times \mathrm{T} \times \mathrm{Ca} \div \mathrm{Cv}+1) \quad \text { para } \quad 0 \leq \mathrm{T} \leq 0,08(\mathrm{Cv} \div \mathrm{Ca})
$$




$$
\begin{array}{lll}
\mathrm{Sa}(\mathrm{T})=2,5 \times a g s 0 & \text { para } & 0,08(\mathrm{Cv} \div \mathrm{Ca}) \leq \mathrm{T} \leq 0,4(\mathrm{Cv} \div \mathrm{Ca}) \\
& \mathrm{Sa}(\mathrm{T})=\operatorname{ags} 1 \div \mathrm{T} & \text { para } \quad \mathrm{T} \geq 0,4(\mathrm{Ca} \div \mathrm{Cv})
\end{array}
$$

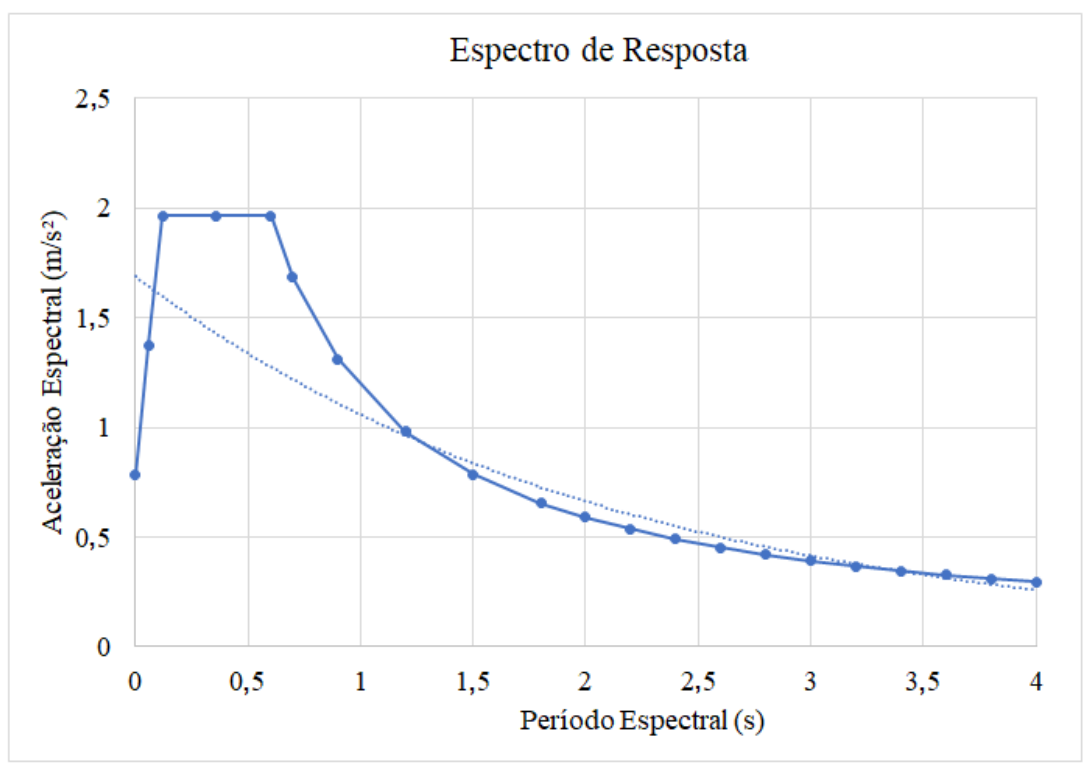

Figura 4: Espectro de Resposta de Projeto

Caracterizado a relação inicial que estabelece o espectro de resposta, constatou-se uma aceleração inicial de projeto com intensidade de $0,780 \mathrm{~m} / \mathrm{s}^{2}$ e acréscimo constante até $1,964 \mathrm{~m} / \mathrm{s}^{2}$, onde se mantem constante durante os períodos de 0,12 a 0,6 segundos, configurando a intensidade máxima de aceleração horizontal de projeto que o modelo está submetido. Posterior a isso, o comportamento apresentado pela relação aceleração-período é a redução crescente das acelerações atuantes.

\subsection{Deslocamentos Totais}

Com a identificação das propriedades mecânicas e caracterização do espectro, tornou-se possível extrair resultados do modelo numérico. Nesse sentido, vê-se a importância de compreender as manifestações de deslocamentos da estrutura como variáveis relacionadas a parâmentos de desempenho e segurança dos mecanismos estruturais atuantes na edificação. Para isso, foram associados os eixo X, Y e Z aos sentidos longitudinal, transversal e vertical, para a obtenção dos deslocamentos direcionais e totais referentes a ações sísmicas introduzidas ao modelo, que são apresentadas na Tabela 3.

Tabela 3 - Deslocamentos obtidos por excitação sísmica do modelo com unidade em metros

\begin{tabular}{c|c|c}
\hline Deslocamentos & Eixo Transversal & Eixo Longitudinal \\
\hline Direcional X & 0,00178 & 0,00191 \\
\hline Direcional Y & 0,00398 & 0,00121 \\
\hline Direcional Z & 0,00040 & 0,00016 \\
\hline Totais & 0,00400 & 0,00190 \\
\hline
\end{tabular}

No que tange os deslocamentos direcionais, presume-se que a exitações promovam maiores deformadas em seus respectivos eixos solicitados, com considerações que abragem eixos ortogonais com magnitudes de resultados que exprimem, sobretudo o comportamento global dessa edificação.

Pode-se tomar como exemplo a relação entre as soliticações de espectro apresentadas no eixo trasversal, com uma deslocamento direcional no eixo Y de 0,00398 metros, associada a uma solicitação no eixo longitudinal de 0,00178 metros, ao passo que as solicitações atuantes no eixo longitudinal apresentaram deslocamentos de 0,00191 metros no respectivo eixo e 0,00121 metros no transversal. Esse fato evidencia uma maior capacidade de solicitação global para as ações sísmicas atuantes no eixo longitudinal, ou X, do modelo. Sob uma perspectiva análoga, pode-se dizer que as solicitações atuantes no eixo transversal manifestaram deslocamentos com magnitude de aproximadamente $44,7 \%$ no eixo ortogonal, a medida que as solicitações no eixo horizontal manifestam $63,3 \%$. 
Destaca-se também as máximas intensidades obtidas, que evidenciam as ações no eixo tranversal da edificação, apontando as alvenarias laterais como de maior susceptibilidade a danos. Nesse sentido, os deslocamentos totais atingiram intensidade de 0,0040 metros sob atuação de ações locais, com predominância de solicitação de flexão nessas regiões. Análogo a isso, o eixo longitudinal apresentou deslocamentos totais de até 0,0019 metros em regiões superiores das alvenarias transversais responsáveis pela divisão dos vãos da edificação, também sob ações de flexão.

Esse fato pode ser atrelado a variáveis como a geometria das alvenarias, que conta com um com maior comprimento efetivo e dimensão livre quando comparada com as alvenarias solicitadas no eixo longitudinal e a presença de arcos capazes de reduzir a rigidez e redirecionar os esforços atuantes.

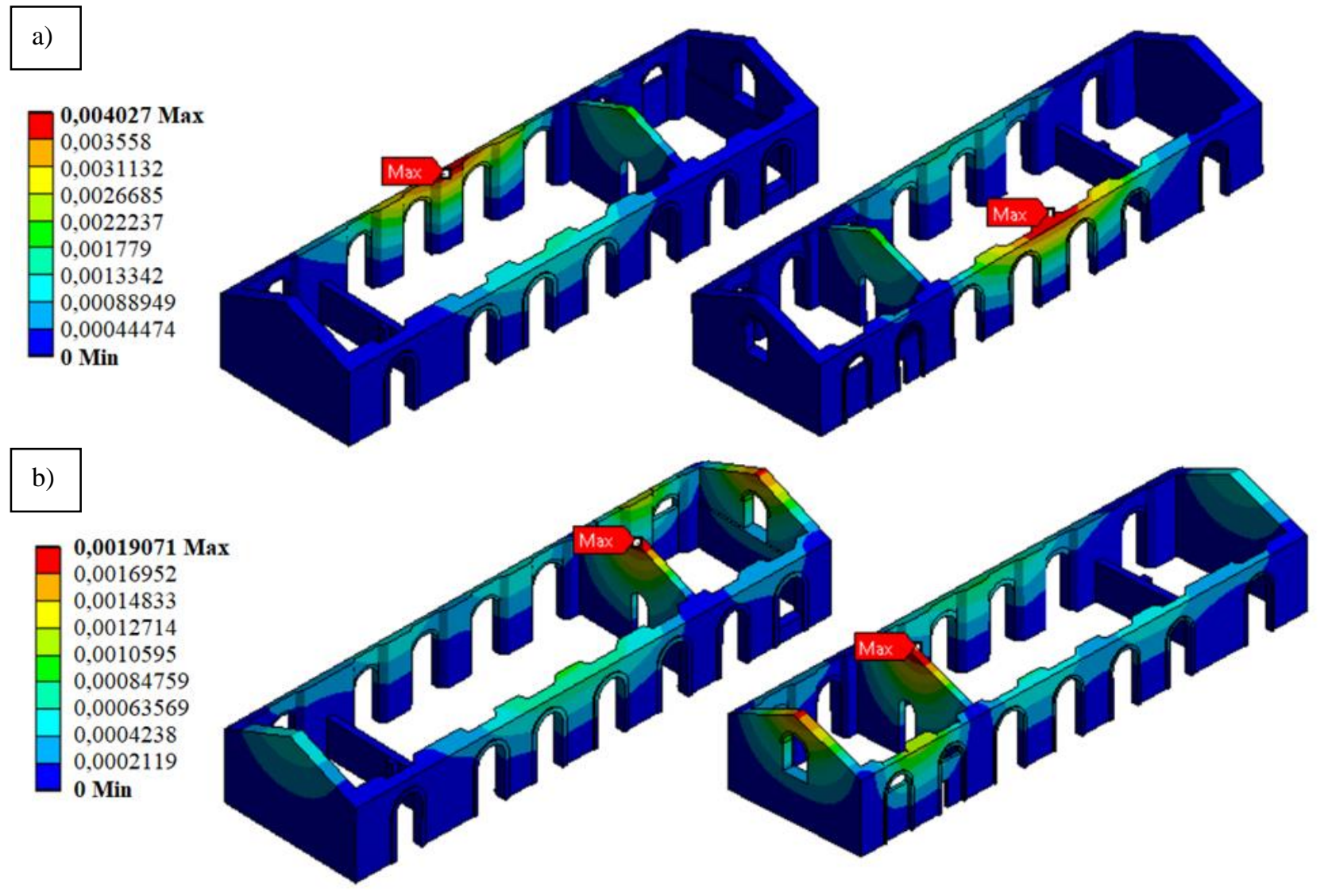

Figura 5: Deslocamentos totais referentes aos eixos transversal (a) longitudinal (b), com unidade em metros.

\subsection{Tensões Equivalentes de Von Mises}

O Critério de Von Mises, também conhecido como Teoria da Energia de Distorção, relaciona as distorções que um material sofre ao acumular energia, no qual o limite de escoamento é atingido quando a quantidade de energia por volume de material se iguala ao limite necessário para o escoamento. Logo, esses conceitos percebidos por Mises e Hencry mediante a experimentos, podem ser expressos em formulações para quantificar a tensão equivalente ( $\sigma$ eq), considerando as tensões atuantes nos planos ortogonais $(\sigma 1, \sigma 2$ e $\sigma 3)$.

$$
\sigma e q=0,5 \sqrt{(\sigma 1-\sigma 2)^{2}+(\sigma 1-\sigma 3)^{2}+(\sigma 3-\sigma 1)^{2}}
$$

Na Figura 6 é possível perceber que o eixo transversal, ao ser solicitado, apresentou tensões superiores ao eixo longitudinal da edificação. Logo, com intensidade de aproximadamente 0,733 MPa, nota-se o acúmulo de tensões de tração em regiões centrais e inferiores, próximas aos arcos que compõem as alvenarias laterais. Análogo a isso, o segundo eixo apresentou também acúmulo de tensões em regiões de união de alvenarias, com magnitudes de até 0,411 MPa. 
É válido salientar que as tensões obtidas são superiores aos limites característicos de tração atribuídos para as alvenarias de pedra e elementos cerâmicos, de 0,24 MPa e 0,16 MPa respectivamente. Desse modo, evidencia-se a possibilidade do surgimento de danos nessas regiões e o potencial das análises estruturais via elementos finitos como uma ferramenta investigativa em prol da manutenção da seguridade e preservação de edificações históricas.

Também foi verificado que os resultados apontam a existência de tensões de compressão com intensidade de 422,68 e 278,81 Pascal, referentes as excitações promovidas para os eixos transversal e longitudinal, respectivamente. Ademais, o modelo apresentou capacidade de absorção desses esforços, não apontando fragilidades no desenvolvimento das simulações.

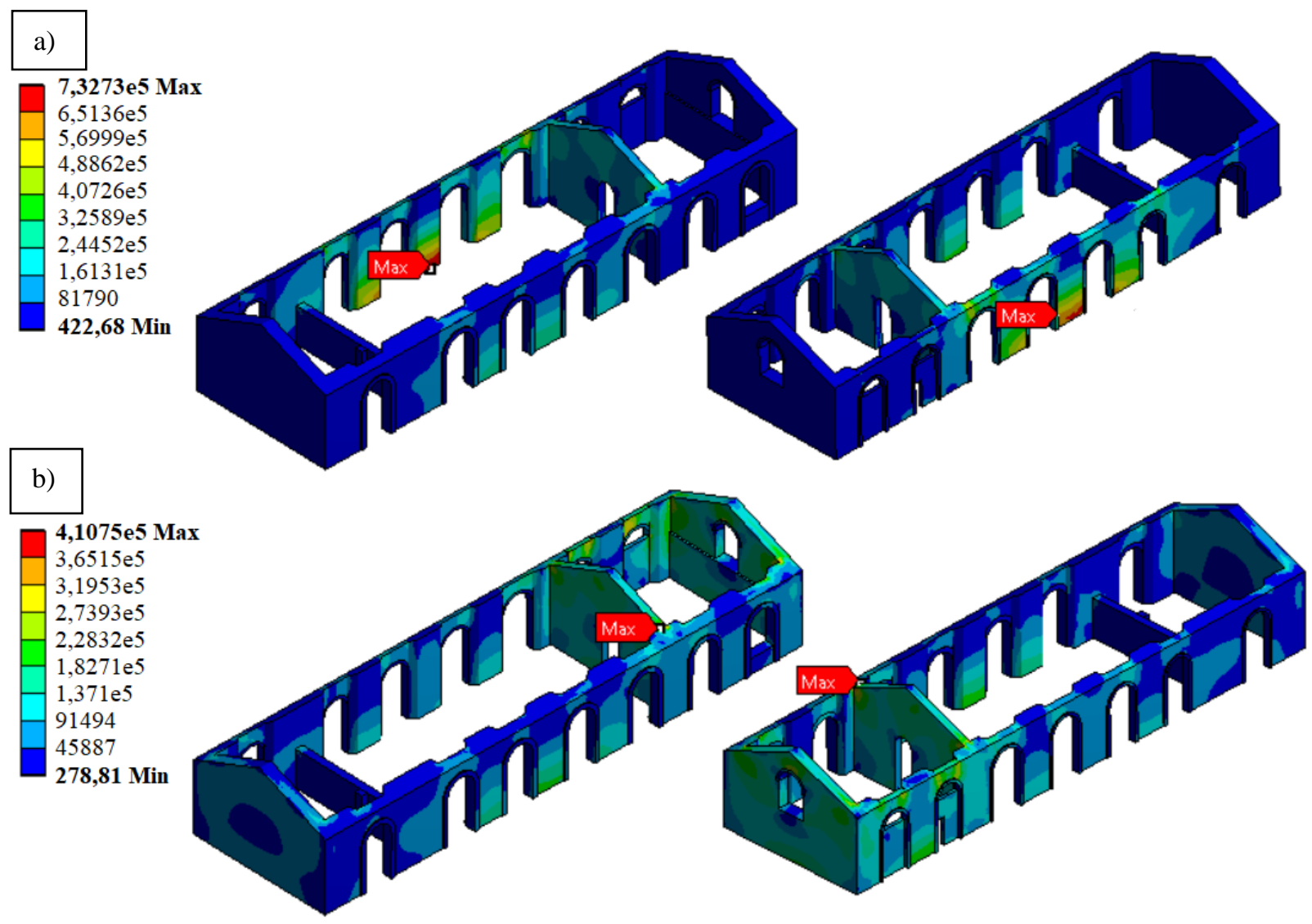

Figura 6: Tensões equivalentes referentes aos eixos transversal (a) longitudinal (b), com unidade em Pascal.

\section{CONCLUSÃO}

Reconhecida a importância das edificações que compõem o patrimonio histórico nacional, é irrefutável a necessidade de desenvolver pesquisas semelhantes, que englobem parâmetros que maximizem a compreensão dos mecanismos estruturais e manifestações danosas que acometem esses bens, para que seja viável o desenvolvimento de atividades de preservação com o máximo de efetividade. Trabalhos dessa natureza são hábeis de promover uma significativa redução de custos associadas a processos inteventivos, maior confiabilidade durante as tomadas de decições, e consequentemente, funcionamento atentendo os parâmetros de desempenho e seguridade.

O estudo de caso abordou o desempenho da edificação frente ações sísmicas provenientes da caracterização do espectro de resposta. Os resultados relacionaram os deslocamentos de maior intensidade as exitações no eixo transversal, com magnitude de 0,004 metros e localização próxima aos arcos que compõem as alvenarias laterais. Frente a isso, deslocamentos de 0,0019 metros foram registradas para o eixo longitudinal, bem como, o eixo apresentou maior capacidade de solicitação global da estrutura.

No tocante das tensões equivalentes, vê-se que as intensidades obtidas em simulação ultrapassam os valores característicos que foram atribuídos ao material. Para as ações nos eixos transversal e longitudinal, obteve-se concentrações de tensões com valores de até 0,733 Mpa e 0,411 Mpa, respectivamente, ambos superiores aos valores de 
0,24 MPa e 0,16 MPa que foram atribuídos para elementos de pedra e cerâmicos. Ainda com destaque das alvenarias laterais da edificação, acrescidas de interseções entre alvenarias, associa-se o fato aos elevados vãos e alturas que compõem a edificação e ao redirecionamento de esforços devido a presença de arcos ao longo do eixo longitudinal.

Os resultados muito informaram a respeito do comportamento global da edificação, possibilitando quantificar e localizar deslocamentos e tensões atuantes nos painéis de alvenaria. As informações obtidas com uso de simulações computacionais via Método dos Elementos Finitos permitiram identificar possíveis regiões de danos, mostrando-se como uma alternativa eficaz para a preservação do patrimônio histórico, apta a informar sobre o comportamento estrutural, sobretudo no que trata da previsão de danos.

\section{REFERÊNCIAS}

ASSOCIAÇÃO BRASILEIRA DE NORMAS TÉCNICAS. NBR 6120: Cargas para o cálculo de estruturas de edificações. Rio de Janeiro, 1980.

ASSOCIAÇÃO BRASILEIRA DE NORMAS TÉCNICAS. NBR 15421: Projeto de estruturas resistentes a sismos Procedimento, Brasil, 2006.

BRANCO, M. E. M. Reforço Sísmico de Edifícios de Alvenaria -Aplicação a edifícios "Gaioleiros". 2007.98 f. Dissertação (Mestrado em Engenharia Civil) -Instituto Superior Técnico, Lisboa, 2007.

BRANDÃO, F. Análise do Comportamento Sísmico de uma Construção Histórica do Patrimônio Sobralense. Monografia. Universidade Estadual Vale do Acarau -UVA, Sobral, 2017.

CARTA DE CRACÓVIA. Princípios para a conservação e o restauro do património construído. 2000. Disponível em: < http://www.patrimoniocultural.gov.pt/media/uploads/cc/cartadecracovia2000.pdf >. Acesso em: 05 jan. 2020.

FRAZÃO, Maria Tereza de Brito Rações Franco. Modelação de um edifício “Gaioleiro" para Avaliação e Reforço Sísmico. Dissertação de Mestrado. Instituto Superior Técnico de Lisboa, Portugal, 2013.

NEVES, S. M. L. C. Análise Sísmica de um Edifício da Baixa Pombalina. 2008. 123 f. Dissertação (Mestrado em Engenharia Civil) -Instituto Superior Técnico, Lisboa, 2008. Disponível em: < https://fenix.tecnico.ulisboa.pt/downloadFile/395137661650/An\%C3\%A1lise\%20S\%C3\%ADsmica\%20de\%20um\%20 Edif\%C3\%ADcio\%20da\%20Baixa\%20Pombalina\%20-\%20S\%C3\%ADlvia\%20Neves.pdf> Acesso em: 8 jan. 2020.

LOURENÇO, P. B. Computations on historic masonry structures. Progress in structural engineering and materials, v. 4, n.3, p. 301-319, jul/sep. 2002.

MINISTERO DELLE INFRASTRUTTURE E DEI TRASPORTI. Norme Tecniche per le Costruzioni (NTC 2018) D.M. del 14/01/2008. Roma, 2018.

NUNES, Ricardo Filipe Gomes. Modelação e análise de estruturas de edifícios existentes em alvenaria. 2013. Tese de Doutorado. Instituto Politécnico de Tomar. Disponível em: <https:// https://comum.rcaap.pt/handle/10400.26/5822> Acesso em: 8 jan. 2020.

PELETEIRO, Suzana Campana. Contribuições à Modelagem Numérica de Alvenaria Estrutural. Tese de Doutorado em Engenharia de Estruturas, Universidade de São Paulo. 2002.

PEREIRA, A. Q. O centro urbano de Quixadá. In: Anais do V Congresso de Pesquisa e Inovação da Rede Norte Nordeste de Educação Tecnológica, 2010, Maceió. v. I. p. 1-7.

SILVA, CLÁUDIO ANTÔNIO VIEIRA DA. Há "pedras" no meu curral: a paisagem dos monólitos de QuixadáCE, 2017. 195 fls. Dissertação (Mestrado em Preservação do Patrimônio Cultural) - IPHAN, Rio de Janeiro, 2017.

ROCA, Pere et al. Structural analysis of masonry historical constructions. Classical and advanced approaches. Archives of Computational Methods in Engineering, v. 17, n. 3, p. 299-325, 2010. 